\title{
Transtornos mentais comuns em docentes do ensino superior: evidências de aspectos sociodemográficos e do trabalho
}

\author{
Common mental disorders in higher education teachers: \\ evidence of sociodemographical and work aspects
}

\begin{abstract}
Taís Cordeiro Campos ${ }^{1}-{ }^{1}$ Universidade Federal do Recôncavo da Bahia | Centro de Ciências Agrárias, Ambientais e Biológicas | Cruz das Almas | BA | Brasil. Contato: taiccampos@gmail.com. ORCID: https://orcid.org/0000-0002-0265-9701
\end{abstract}

Renata Meira Véras ${ }^{2}{ }^{2}$ Universidade Federal da Bahia | Instituto de Humanidades, Artes e Ciências Milton Santos | Salvador | BA | Brasil. Contato: renatameiraveras@gmail.com. ORCID: https://orcid.org/0000-0002-1681-1401

Tânia Maria de Araújo ${ }^{3}$ - ${ }^{3}$ Universidade Estadual de Feira de Santana | Departamento de Saúde | Feira de Santana | BA | Brasil. Contato: araujo.tania@uefs.br. ORCID: https://orcid.org/0000-0003-2766-7799

Resumo: Os Transtornos Mentais Comuns (TMC) apresentaram crescimento significativo nas últimas décadas. Apesar das elevadas prevalências registradas e de representar importante causa de afastamento do trabalho entre docentes, ainda há lacunas do conhecimento sobre esse evento em docentes universitários. Este estudo objetivou estimar a prevalência de TMC em docentes de uma universidade pública da Bahia, analisando sua associação com aspectos sociodemográficos, laborais e psicossociais. Foi realizado estudo epidemiológico de corte transversal, de caráter exploratório, envolvendo 127 docentes. Foram realizadas análises univariada, bivariada e multivariada com regressão logística não condicional. Para entrada no modelo, estabeleceu-se valor de $\mathrm{p} \leq 0,25$, obtido pelo Teste Qui-Quadrado de Pearson $\left(\mathrm{X}^{2}\right)$ ou Exato de Fisher. As razões de prevalência foram estimadas por Regressão de Poisson. O nível de significância estatística adotado para permanência no modelo final foi de 5\%. A prevalência de TMC na população estudada foi de $29,9 \%$. Observou-se associação estatisticamente significante de TMC com sentimento de desgaste na relação com os alunos (RP: 2,31) e falta de satisfação em trabalhar na instituição (RP: 2,13). Elevada prevalência de TMC foi observada entre os docentes participantes do estudo reforçando a necessidade de ações de intervenção e do olhar atento sobre a saúde mental destes profissionais.

Palavras-chave: Docentes. Estudos Transversais. Transtornos mentais.

Abstract: Common Mental Disorders (CMD) have grown significantly in recent decades. Despite the high prevalences recorded and representing an important cause of work leave among teachers, there are still gaps in knowledge about this event among university professors. This study aimed to estimate the prevalence of CMD among professors of a public university in Bahia, analyzing its association with sociodemographic, labor and psychosocial aspects. An exploratory cross-sectional epidemiological study was carried out involving 127 teachers. Univariate, bivariate and multivariate analyzes were performed with unconditional logistic regression. For entry into the model, a value of $\mathrm{p} \leq 0,25$ was obtained, obtained by Pearson's Chi-Square Test $\left(\mathrm{X}^{2}\right)$ or Fisher's Exact. Prevalence ratios were estimated by Poisson Regression. The level of statistical significance adopted for permanence in the final model was $5 \%$. The prevalence of CMD in the study population was $29,9 \%$. There was a statistically significant association of CMD with feeling of weariness in the relationship with the students (PR: 2,31) and lack of satisfaction in working in the institution (PR: 2,13). High prevalence of CMD was observed among the teachers participating in the study, reinforcing the need for intervention actions and a close look at the mental health of these professionals.

Keywords: Faculty. Cross-sectional studies. Mental disorders.

- Recebido em: 25 de outubro de 2019 - Aprovado em: 25 de junho de 2020

DOI: http://dx.doi.org/10.1590/S1414-40772020000300012

Este é um artigo publicado em acesso aberto sob uma licença Creative Commons https://creativecommons.org/licenses/by-nc/4.0/ 


\section{Introdução}

Os transtornos mentais estão entre os principais problemas de saúde que afetam os docentes (ARAÚJO; CARVALHO, 2009). Apesar de não serem eventos com impacto mais direto na mortalidade, podem levar a incapacitações graves e definitivas, acarretando redução da qualidade de vida dos indivíduos. São responsáveis por gerar alto custo social e econômico (SANTOS; SIQUEIRA, 2010), uma vez que representam a terceira principal causa de concessão do benefício auxílio-doença por incapacidade laborativa no Brasil (SILVA-JUNIOR; FISCHER, 2015).

Os chamados Transtornos Mentais Comuns (TMC) incluem sintomatologia de depressão, ansiedade e transtornos somatoformes (SANTOS; SIQUEIRA, 2010), tais como insônia, nervosismo, dores de cabeça, fadiga, irritabilidade, esquecimento, dificuldade de concentração e queixas somáticas inespecíficas (LUDERMIR; MELO FILHO, 2002). Segundo Carlotto e Câmara (2015), dentre os distúrbios que caracterizam os TMC, a depressão e a ansiedade são as manifestações de sofrimento mais importantes, do ponto de vista da saúde pública, em razão da frequência com que acometem a população em geral.

Estudos epidemiológicos têm revelado elevadas prevalências de TMC em trabalhadores brasileiros de diversas áreas, incluindo agentes comunitários de saúde, motoristas e cobradores de ônibus, trabalhadores rurais, funcionários de universidades e trabalhadores de enfermagem, variando de 20,3\% a 43,3\% (SANTOS; SIQUEIRA, 2010). Pesquisas envolvendo docentes também evidenciam elevadas frequências desses eventos em todos os níveis de ensino: 17,8\% (SILVA; SILVA, 2013), 21,8\% (LYRA et al., 2009), 41,5\% (DELCOR et al., 2004), 50\% (JARDIM; BARRETO; ASSUNÇÃO, 2007) e 55,9\% (REIS et al., 2005) em docentes do ensino básico; 18,7\% (ARAÚJO; CARVALHO, 2009) e 19,5\% (FERREIRA et al., 2015) em docentes do ensino superior. Observa-se que grande parte das pesquisas tem se dedicado aos docentes do ensino básico (especialmente fundamental e médio), sendo menos frequentes as pesquisas envolvendo docentes do ensino superior, embora os dados existentes evidenciem também a sua importância nesse grupo.

Diversos aspectos podem atuar conjuntamente no desencadeamento dos transtornos mentais (GLINA et al., 2001), sejam eles vinculados especificamente ao mundo do trabalho, ou fora dele. Condições de vida e trabalho desfavoráveis, falta de reconhecimento, dificuldades na vida familiar, problemas de comportamento dos alunos e falta de acompanhamento por parte da família são fatores que podem estar relacionados ao adoecimento mental em docentes (DIEHL; MARIN, 2016; LYRA et al., 2009). 
Dentre os fatores associados, destaca-se a importância da organização do trabalho, conforme proposto pelas teorias da psicodinâmica do trabalho e do estresse ocupacional (GLINA et al., 2001). Desta forma, grande parte dos estudos sobre o adoecimento mental em docentes tem abordado a influência do trabalho (LYRA et al., 2009). Relações com colegas de trabalho conflituosas, trabalho repetitivo, ritmo acelerado de trabalho (ARAÚJO et al., 2003; DELCOR et al., 2004; REIS et al., 2005), volume excessivo de atividades, tempo para realização das tarefas insuficiente (DELCOR et al., 2004; REIS et al., 2005), sobrecarga (CARLOTTO; CÂMARA, 2015) são alguns dos aspectos que se mostraram associados aos TMC em docentes, de acordo com pesquisas epidemiológicas. Assim, as variáveis psicossociais revelam-se fatores importantes para a ocorrência dos TMC em docentes (CARLOTTO; CÂMARA, 2015).

Diante dos poucos estudos envolvendo docentes universitários de instituições públicas e considerando as elevadas prevalências de TMC em docentes, assim como sua associação com aspectos do trabalho, torna-se pertinente a realização de pesquisas que investiguem tal temática. Neste sentido, o presente estudo visa estimar a prevalência de TMC em docentes de uma universidade pública da Bahia, analisando sua associação com características sociodemográficas, laborais e psicossociais.

\section{Métodos}

Esta pesquisa consiste em estudo epidemiológico de corte transversal, de caráter exploratório, envolvendo docentes de uma universidade pública da Bahia. A universidade estudada foi instituída em um modelo de multicampia sendo formada por sete unidades distribuídas em seis municípios (Amargosa, Cachoeira, Cruz das Almas, Santo Antônio de Jesus, Feira de Santana e Santo Amaro). Do conjunto de unidades que compõe a instituição, foi selecionada aquela com maior quantidade de docentes e de cursos de graduação e pósgraduação. À época da pesquisa havia 170 docentes distribuídos por nove cursos de graduação (Agronomia, Bacharelado e Licenciatura em Biologia, Engenharia Florestal e de Pesca, Medicina Veterinária, Tecnologia em Agroecologia e em Gestão de Cooperativas e Zootecnia) e onze cursos de pós-graduação (uma especialização Lato Sensu, seis mestrados acadêmicos, dois mestrados profissionais e dois doutorados acadêmicos).

Buscou-se realizar um censo dos docentes lotados na unidade selecionada. Foram elegíveis para a pesquisa todos os docentes efetivos lotados na unidade escolhida, em exercício profissional há pelo menos um ano na instituição e em plena atividade nos trinta dias que 
antecederam o período da coleta dos dados. A lista de docentes atualizada foi fornecida pelo setor de gestão de pessoal contendo 159 docentes elegíveis. Destes, houve uma perda de 32 docentes, equivalendo a 20,1\% daqueles elegíveis: oito estavam afastados para estudo, quatro estavam em licença gestante, dois estavam em licença para tratamento da saúde, dez não foram localizados em seus gabinetes de trabalho, sete não devolveram o questionário preenchido e um docente se recusou a participar. Desta forma, a população estudada foi composta por 127 docentes, equivalendo a 79,9\% dos docentes elegíveis.

A coleta de dados foi realizada no período referente ao meio do semestre, de novembro a dezembro de 2017. Os docentes foram visitados em seus gabinetes de trabalho, momento em que foram explicados os objetivos da pesquisa e consultados sobre a possibilidade de participação. Após o aceite e assinatura do Termo de Consentimento Livre e Esclarecido, o questionário foi entregue em envelope individual ao docente e, após seu preenchimento, foi coletado em envelope lacrado sem identificação. Os docentes foram procurados em seus locais de trabalho nos turnos diurno e noturno durante cinco dias da semana (segunda a sexta-feira). Os docentes não localizados foram procurados em diferentes turnos e dias da semana até o momento de finalização da coleta dos dados.

Para a coleta dos dados foi utilizado questionário semiestruturado, autoaplicável, composto por seis blocos de questões: Bloco A - Perfil Sociodemográfico; Bloco B - Perfil Profissional; Bloco C - Características do Trabalho; Bloco D - Aspectos Psicossociais do Trabalho; Bloco E - Hábitos de Vida; Bloco F - Saúde Mental. O questionário utilizado nesta pesquisa foi adaptado de um instrumento testado e aplicado por Santos (2016) em estudo realizado com docentes da Universidade Estadual de Feira de Santana (UEFS).

As variáveis independentes utilizadas no estudo foram: sociodemográficas (sexo, idade, filhos, situação conjugal e local de origem); laborais (titulação máxima, tempo de trabalho como docente na instituição estudada, carga horária semanal (na graduação, pós-graduação, em atividades de pesquisa, extensão e administrativas), carga horária semanal total (em atividades de ensino, pesquisa, extensão e administrativas, concomitantemente), sentimento de sobrecarga de trabalho, pressão por publicação, desgaste na relação com os alunos, sentimento de satisfação em trabalhar na universidade, pensamento de abandonar a instituição, condições da sala de aula, história de agressão física ou verbal sofrida dentro do campus e sentimento de segurança dentro do campus); e aspectos psicossociais do trabalho (demanda psicológica e controle sobre o próprio trabalho, extraídas do questionário Job Content Questionnaire - JCQ).

A variável "condições da sala de aula" corresponde a um indicador criado com base no somatório das variáveis iluminação, acústica, ruído, temperatura, condições de higiene, 
tamanho das salas de aula, condições de cadeiras e mesas e recursos audiovisuais. Em seguida, tal indicador foi categorizado em tercis: condições da sala de aula adequada, regular ou inadequada.

O JCQ é um instrumento desenvolvido para avaliar aspectos psicossociais do trabalho. Sua versão recomendada contêm 49 questões que abordam seis dimensões: controle sobre o próprio trabalho, demanda psicológica, demanda física, apoio social no trabalho, insegurança no emprego e nível educacional exigido (ARAÚJO; GRAÇA; ARAÚJO, 2003). Neste estudo foram utilizadas apenas duas dimensões: demanda psicológica (5 itens) e controle sobre o trabalho (6 itens sobre habilidade e 3 itens sobre autoridade de decisão). As questões do JCQ apresentam-se dispostas em escala do tipo likert de quatro pontos (discordo fortemente/ discordo/ concordo/ concordo fortemente).

O instrumento JCQ baseia-se no modelo Demanda-Controle (D-C), elaborado por Robert Karasek, em 1979. Tal modelo busca relacionar a demanda psicológica envolvida na execução do trabalho e o controle sobre o próprio trabalho com as repercussões sobre a estrutura psíquica e orgânica dos trabalhadores. A combinação de diferentes níveis de demanda e controle possibilita a caracterização de situações específicas de trabalho, constituídas em quatro categorias: baixa exigência do trabalho (baixa demanda psicológica e alto controle sobre o trabalho), trabalho ativo (alta demanda e alto controle), trabalho passivo (baixa demanda e baixo controle) e alta exigência (alta demanda e baixo controle) (ARAÚJO; GRAÇA; ARAÚJO, 2003; PORTO et al., 2006; REIS et al., 2005).

Para a construção das situações de trabalho, inicialmente calculou-se o valor da demanda psicológica e do controle sobre o trabalho aplicando-se a fórmula proposta pelo modelo e disponível no endereço eletrônico do JCQ Center (www.JCQCenter.org). A partir dos escores obtidos, cada dimensão foi dicotomizada em "alta" e "baixa" utilizando como ponto de corte a mediana do valor de cada dimensão do modelo. Desta forma, por meio da combinação dos diferentes níveis de demanda psicológica e de controle sobre o trabalho foram constituídas as quatro experiências de trabalho propostas pelo modelo. $\mathrm{O}$ trabalho caracterizado como de alta exigência foi classificado como grupo de maior exposição, enquanto que o trabalho caracterizado como de baixa exigência foi considerado como grupo de referência, seguindo os pressupostos do modelo D-C.

O Bloco F refere-se à variável dependente do estudo e consiste na avaliação da presença de TMC por meio do Self-Reporting Questionnaire-20 (SRQ-20). O SRQ-20 visa detectar transtornos mentais não psicóticos, sendo recomendado pela Organização Mundial da Saúde para estudos comunitários e em atenção básica à saúde por ser de baixo custo e fácil aplicação 
(GONÇALVES; STEIN; KAPCZINSKI, 2008). É um instrumento bastante utilizado e validado em diversos países, inclusive no Brasil, que demonstra boa habilidade para identificar TMC na atenção primária à saúde (GONÇALVES; STEIN; KAPCZINSKI, 2008; SANTOS et al., 2010). Seu desempenho em rastrear TMC foi avaliado em trabalhadores, sendo considerado aceitável na investigação da saúde mental em âmbito ocupacional (SANTOS; ARAÚJO; OLIVEIRA, 2009).

O SRQ-20 é composto por 20 questões que avaliam quatro grupos de sintomas: presença de humor depressivo-ansioso, sintomas somáticos, decréscimo de energia vital e pensamentos depressivos. O questionário deve ser respondido com base na presença desses sintomas nos últimos 30 dias (SANTOS; ARAÚJO; OLIVEIRA, 2009). As questões do SRQ-20 são do tipo dicotômicas ( $\operatorname{sim} /$ não) pontuadas em 1 ou 0 . A pontuação 1 indica que o sintoma esteve presente nos últimos trinta dias, enquanto que a pontuação 0 indica que o sintoma esteve ausente. $\mathrm{O}$ escore é obtido pela soma dos pontos e a pontuação máxima é 20 (BEUSENBERG et al., 1994). O ponto de corte adotado neste estudo foi de sete ou mais respostas positivas como sugestivo de TMC, seguindo indicação de Santos et al. (2010) que, em estudo de avaliação do desempenho do SRQ-20 em população urbana, encontraram como ponto de corte de melhor desempenho o escore sete. Tal escore foi adotado também em outros estudos envolvendo docentes universitários (FIGLIOULO; LIMA; LAURENTINO, 2011; TAVARES et al., 2012).

A análise dos dados se deu por meio de estatística descritiva. Para tanto, foram realizadas análises univariada, bivariada e multivariada. Inicialmente foi realizada a caracterização da população investigada, estimando-se as frequências relativa e absoluta de cada variável estudada. Em seguida, foi estimada a prevalência global dos TMC. Na análise bivariada foram estimadas as prevalências de TMC e seus respectivos intervalos de confiança (IC a 95\%). Nestas duas primeiras análises foi utilizado o software SPSS (Statical Package for the Social Science, versão 22.0).

Na pré-seleção das variáveis para a análise multivariada foi empregado o teste QuiQuadrado de Person $\left(\mathrm{X}^{2}\right)$ ou Exato de Fisher, adotando-se nível de significância de $\mathrm{p} \leq 0,25$ para entrada das variáveis no modelo multivariado. Para a análise multivariada foi utilizada a regressão logística não condicional. Foi aplicado o modelo de regressão logística multivariada, a fim de verificar os fatores associados aos TMC, utilizando-se o software STATA (Data Analysis and Statistical Softwares, versão 12.0). As razões de prevalência (RP) foram estimadas utilizando Regressão de Poisson com variação robusta. O nível de significância estatística adotado como critério para permanência das variáveis no modelo final foi o valor de $\mathrm{p} \leq 0,05$, utilizando-se o método Backward para a seleção das variáveis. Por fim, para análise diagnóstica 
do modelo final foi calculada a área sob a curva ROC. Além disso, avaliou-se o grau de acurácia e qualidade do ajuste do modelo por meio do teste de Hosmer-Lemeshow.

Esta pesquisa cumpriu os requisitos éticos sendo aprovada pelo Comitê de Ética em Pesquisa da Escola de Enfermagem da Universidade Federal da Bahia em 13 de setembro de 2017. Está registrada sob o número do Certificado de Apresentação para Apreciação Ética: 70803917.4.0000.5531.

\section{Resultados}

\subsection{Caracterização da amostra}

Na população estudada, observou-se que a maioria dos docentes era do sexo masculino $(55,1 \%)$, tinha entre 30 e 45 anos $(54,4 \%)$ com idade média de 46 anos, com filhos $(67,7 \%)$, casado(a) ou possuía união estável $(71,7 \%)$ e com local de origem de um estado diferente da Bahia (57,3\%) (Tabela 1).

Tabela 1 - Distribuição (\%) dos docentes segundo características sociodemográficas. Docentes universitários, Bahia, 2017

\begin{tabular}{lcc}
\hline Características Sociodemográficas (N) & $\mathrm{n}$ & $\%$ \\
\hline Sexo (127) & 57 & 44,9 \\
Mulheres & 70 & 55,1 \\
Homens & & \\
Idade (125) & 68 & 54,4 \\
Entre 30 e 45 anos & 57 & 45,6 \\
Entre 46 e 67 anos & & \\
Tem Filhos (127) & 86 & 67,7 \\
Sim & 41 & 32,3 \\
Não & 36 & 28,3 \\
Situação Conjugal (127) & 91 & 71,7 \\
Sem companheiro* & & \\
Com companheiro* & 53 & 42,7 \\
Local de Origem (124) & 71 & 57,3 \\
Bahia & & \\
Outros** & & \\
\hline
\end{tabular}

Fonte: banco de dados

* Sem companheiro= solteiro, viúvo, divorciado / Com companheiro = casado, união estável

** Alagoas, Cabo Verde, Caracas, Ceará, Distrito Federal, Espírito Santo, Goiás, Maranhão, Minas Gerais, Paraíba, Paraná, Pernambuco, Rio de Janeiro, Rio Grande do Norte, Rio Grande do Sul, São Paulo, Sergipe

Considerando as características laborais (Tabela 2), todos os docentes possuíam elevado nível de titulação, mestrado ou doutorado, destacando-se elevado percentual com doutorado $(95,2 \%)$. Quanto à carga horária total referente às atividades de ensino (graduação e pósgraduação), pesquisa, extensão e administrativas, realizadas concomitantemente, a maioria dos 
docentes $(59,8 \%)$ dedicava até 30 horas por semana. Quase dois terços dos docentes referiram sentir sobrecarga no desempenho das atividades laborais $(65,4 \%$,) e pressão pela produção e publicação de artigos $(66,1 \%)$. Condições da sala de aula adequadas foram referidas por 41,6\%. Quando indagados sobre desgaste na relação com os alunos, 73,2\% referiram não sentir desgaste; no entanto, uma parcela significativa (43,3\%) relatou já ter sofrido alguma agressão, física ou verbal, de alunos, colegas ou pessoas externas, dentro da instituição e, neste cenário, a maioria dos docentes, $62,2 \%$, relatou não sentir segurança ou proteção na universidade. Estar satisfeito em trabalhar na instituição foi relatado por 71,7\%; e 55,9\% afirmaram não desejar abandonar a universidade estudada. 
Tabela 2 - Distribuição (\%) dos docentes segundo características laborais. Docentes universitários, Bahia, 2017

\begin{tabular}{|c|c|c|}
\hline Características Laborais (N) & $\mathrm{n}$ & $\%$ \\
\hline \multicolumn{3}{|l|}{ Titulação Máxima (126) } \\
\hline Mestrado & 6 & 4,8 \\
\hline Doutorado & 120 & 95,2 \\
\hline \multicolumn{3}{|c|}{ Tempo de Docência na Instituição (126) } \\
\hline Entre 1 e 7 anos & 53 & 42,1 \\
\hline Acima de 8 anos & 73 & 57,9 \\
\hline \multicolumn{3}{|c|}{ Carga Horária na Graduação (125) } \\
\hline Entre 4 e 12 horas & 100 & 80,0 \\
\hline Acima de 13 horas & 25 & 20,0 \\
\hline \multicolumn{3}{|c|}{ Carga Horária na Pós-Graduação (64) } \\
\hline Entre 1 e 4 horas & 28 & 43,8 \\
\hline Acima de 5 horas & 36 & 56,2 \\
\hline \multicolumn{3}{|l|}{ Carga Horária de Pesquisa (115) } \\
\hline Entre 1 e 8 horas & 56 & 48,7 \\
\hline Acima de 9 horas & 59 & 51,3 \\
\hline \multicolumn{3}{|l|}{ Carga Horária de Extensão (74) } \\
\hline Entre 1 e 8 horas & 63 & 85,2 \\
\hline Acima de 9 horas & 11 & 14,8 \\
\hline \multicolumn{3}{|c|}{ Carga Horária de Atividades Administrativas (90) } \\
\hline Entre 1 e 4 horas & 61 & 67,8 \\
\hline Acima de 5 horas & 29 & 32,2 \\
\hline \multicolumn{3}{|l|}{ Carga Horária Total (127) } \\
\hline Entre 9 e 30 horas & 76 & 59,8 \\
\hline Acima de 31 horas & 51 & 40,2 \\
\hline \multicolumn{3}{|l|}{ Sentimento de Sobrecarga (127) } \\
\hline Sim & 83 & 65,4 \\
\hline Não & 44 & 34,6 \\
\hline \multicolumn{3}{|l|}{ Pressão por Publicação (127) } \\
\hline Sim & 84 & 66,1 \\
\hline Não & 43 & 33,9 \\
\hline \multicolumn{3}{|c|}{ Desgaste na Relação com Alunos (127) } \\
\hline Sim & 34 & 26,8 \\
\hline Não & 93 & 73,2 \\
\hline \multicolumn{3}{|c|}{ Condições da Sala de Aula (125) } \\
\hline Adequada & 52 & 41,6 \\
\hline Regular & 44 & 35,2 \\
\hline Inadequada & 29 & 23,2 \\
\hline \multicolumn{3}{|c|}{ Episódio de Agressão Dentro do Campus (127) } \\
\hline $\mathrm{Sim}$ & 55 & 43,3 \\
\hline Não & 72 & 56,7 \\
\hline \multicolumn{3}{|c|}{ Sentimento de Proteção no Campus (127) } \\
\hline Sim & 48 & 37,8 \\
\hline Não & 79 & 62,2 \\
\hline \multicolumn{3}{|c|}{ Satisfação em Trabalhar na Universidade (127) } \\
\hline Sim & 91 & 71,7 \\
\hline Não & 36 & 28,3 \\
\hline \multicolumn{3}{|c|}{ Desejo de Abandonar a Instituição (127) } \\
\hline Sim & 56 & 44,1 \\
\hline Não & 71 & 55,9 \\
\hline
\end{tabular}

Fonte: banco de dados

Quanto aos aspectos psicossociais do trabalho (Tabela 3), observou-se que a maioria dos docentes apresentava alta demanda psicológica $(53,2 \%)$ e alto controle sobre o trabalho 
(51,6\%). De acordo com o modelo D-C, o percentual mais elevado das experiências do trabalho foi observado em situação de "alta exigência" $(28,5 \%)$.

Tabela 3 - Distribuição (\%) dos docentes segundo aspectos psicossociais. Docentes universitários, Bahia, 2017

\begin{tabular}{lll}
\hline Aspectos Psicossociais do Trabalho (N) & $\mathrm{n}$ & $\%$ \\
\hline Demanda Psicológica (126) & 59 & 46,8 \\
$\quad$ Baixa & 67 & 53,2 \\
$\quad$ Alta & & \\
Controle Sobre o Trabalho (124) & 64 & 51,6 \\
$\quad$ Alto & 60 & 48,4 \\
$\quad$ Baixo & & 26,0 \\
Modelo D-C (123) & 32 & 25,2 \\
Baixa exigência & 31 & 20,3 \\
Trabalho ativo & 25 & 28,5 \\
Trabalho passivo & 35 & \\
Alta exigência
\end{tabular}

Fonte: banco de dados

\subsection{Prevalência de TMC e fatores associados}

A prevalência global de TMC encontrada na população investigada foi de $29,9 \%$. Na análise bivariada da associação entre TMC e aspectos sociodemográficos, observou-se associação estatisticamente significativa $(\mathrm{p} \leq 0,05)$ para: idade entre 30 e 45 anos; ter filhos; e local de origem outros (Tabela 4). 
Tabela 4 - Prevalência (\%) de TMC, razões de prevalência e respectivos intervalos de confiança de 95\%, segundo características sociodemográficas. Docentes universitários, Bahia, 2017

\begin{tabular}{|c|c|c|c|c|c|}
\hline \multirow{2}{*}{ Características Sociodemográficas $(\mathrm{N})$} & \multicolumn{5}{|c|}{ TMC } \\
\hline & $\mathrm{n}$ & $\%$ & $\mathrm{RP}$ & IC95\% & Valor de $\mathrm{p}$ \\
\hline \multicolumn{6}{|l|}{ Sexo (127) } \\
\hline Mulheres & 18 & 31,6 & 1,11 & $0,649-1,882$ & 0,71 \\
\hline Homens & 20 & 28,6 & --- & --- & --- \\
\hline \multicolumn{6}{|l|}{ Idade (125) } \\
\hline Entre 30 e 45 anos & 25 & 36,8 & 1,75 & $0,967-3,155$ & 0,05 \\
\hline Entre 46 e 67 anos & 12 & 21,1 & --- & --- & --- \\
\hline \multicolumn{6}{|l|}{ Tem Filhos (127) } \\
\hline Sim & 19 & 22,1 & 0,48 & $0,285-0,799$ & $<0,01$ \\
\hline Não & 19 & 46,3 & --- & --- & --- \\
\hline \multicolumn{6}{|l|}{ Situação Conjugal (127) } \\
\hline Sem companheiro & 13 & 36,1 & 1,31 & $0,760-2,273$ & 0,33 \\
\hline Com companheiro & 25 & 27,5 & --- & --- & --- \\
\hline \multicolumn{6}{|l|}{ Local de Origem (124) } \\
\hline Bahia & 11 & 20,8 & --- & --- & --- \\
\hline Outros & 26 & 36,6 & 1,76 & $0,960-3,243$ & 0,05 \\
\hline
\end{tabular}

Fonte: banco de dados

--- = grupo de referência

Quanto às características laborais, foram observadas associações estatisticamente significativas $(\mathrm{p} \leq 0,05)$ para: sentir sobrecarga no trabalho; sentir pressão por publicação; sentir desgaste na relação com os alunos; condição inadequada da sala de aula; não sentir proteção dentro do campus; não sentir satisfação em trabalhar na instituição; e desejo de abandonar a instituição (Tabela 5). 
Tabela 5 - Prevalência (\%) de TMC, razões de prevalência e respectivos intervalos de confiança de 95\%, segundo características laborais. Docentes universitários, Bahia, 2017

\begin{tabular}{|c|c|c|c|c|c|}
\hline \multirow{2}{*}{ Características Laborais (N) } & \multicolumn{5}{|c|}{ TMC } \\
\hline & $\mathrm{n}$ & $\%$ & $\mathrm{RP}$ & IC95\% & Valor de $\mathrm{p}$ \\
\hline \multicolumn{6}{|c|}{ Tempo de Docência na Instituição (126) } \\
\hline Entre 1 e 7 anos & 19 & 35,8 & 1,38 & $0,812-2,336$ & 0,23 \\
\hline Acima de 8 anos & 19 & 26,0 & --- & --- & --- \\
\hline \multicolumn{6}{|c|}{ Carga Horária na Graduação (125) } \\
\hline Entre 4 e 12 horas & 33 & 33,0 & --- & --- & --- \\
\hline Acima de 13 horas & 5 & 20,0 & 0,61 & $0,264-1,393$ & 0,20 \\
\hline \multicolumn{6}{|c|}{ Carga Horária na Pós-Graduação (64) } \\
\hline Entre 1 e 4 horas & 9 & 32,1 & --- & --- & --- \\
\hline Acima de 5 horas & 9 & 25,0 & 0,78 & $0,356-1,698$ & 0,52 \\
\hline \multicolumn{6}{|c|}{ Carga Horária de Pesquisa (115) } \\
\hline Entre 1 e 8 horas & 14 & 25,0 & --- & --- & --- \\
\hline Acima de 9 horas & 22 & 37,3 & 1,49 & $0,851-2,615$ & 0,15 \\
\hline \multicolumn{6}{|l|}{ Carga Horária de Extensão (74) } \\
\hline Entre 1 e 8 horas & 20 & 31,7 & --- & --- & --- \\
\hline Acima de 9 horas & 4 & 36,4 & 1,15 & $0,484-2,711$ & 0,76 \\
\hline \multicolumn{6}{|c|}{ Carga Horária Ativ. Administrativas (90) } \\
\hline Entre 1 e 4 horas & 20 & 32,8 & --- & --- & --- \\
\hline Acima de 5 horas & 9 & 31,0 & 0,95 & $0,494-1,814$ & 0,86 \\
\hline \multicolumn{6}{|l|}{ Carga Horária Total (127) } \\
\hline Entre 9 e 30 horas & 21 & 27,6 & --- & --- & --- \\
\hline Acima de 31 horas & 17 & 33,3 & 1,21 & $0,709-2,054$ & 0,49 \\
\hline \multicolumn{6}{|c|}{ Sentimento de Sobrecarga (127) } \\
\hline Sim & 32 & 38,6 & 2,83 & $1,281-6,240$ & $<0,01$ \\
\hline Não & 6 & 13,6 & --- & --- & --- \\
\hline \multicolumn{6}{|l|}{ Pressão por Publicação (127) } \\
\hline Sim & 30 & 35,7 & 1,92 & $0,965-3,819$ & 0,04 \\
\hline Não & 8 & 18,6 & --- & --- & --- \\
\hline \multicolumn{6}{|c|}{ Desgaste na Relação com Alunos (127) } \\
\hline Sim & 20 & 58,8 & 3,04 & $1,841-5,017$ & $<0,01$ \\
\hline Não & 18 & 19,4 & --- & --- & --- \\
\hline \multicolumn{6}{|c|}{ Condições da Sala de Aula (125) } \\
\hline Adequada & 10 & 19,2 & --- & --- & --- \\
\hline Regular & 14 & 31,8 & 1,66 & $0,817-3,349$ & 0,15 \\
\hline Inadequada & 13 & 44,8 & 2,33 & $1,172-4,638$ & 0,01 \\
\hline \multicolumn{6}{|c|}{ Episódio de Agressão no Campus (127) } \\
\hline Sim & 20 & 36,4 & 1,46 & $0,855-2,474$ & 0,16 \\
\hline Não & 18 & 25,0 & --- & --- & --- \\
\hline \multicolumn{6}{|c|}{ Sentimento de Proteção no Campus (127) } \\
\hline Sim & 7 & 14,6 & --- & --- & --- \\
\hline Não & 31 & 39,2 & 2,69 & $1,287-5,626$ & $<0,01$ \\
\hline \multicolumn{6}{|c|}{ Satisfação em Trabalhar na Instituição (127) } \\
\hline Sim & 19 & 20,9 & --- & --- & --- \\
\hline Não & 19 & 52,8 & 2,53 & $1,525-4,190$ & $<0,01$ \\
\hline \multicolumn{6}{|c|}{ Desejo de Abandonar a Instituição (127) } \\
\hline Sim & 24 & 42,9 & 2,17 & $1,244-3,799$ & $<0,01$ \\
\hline Não & 14 & 19,7 & --- & --- & --- \\
\hline
\end{tabular}

Fonte: banco de dados

--- = valor de referência

Considerando os aspectos psicossociais do trabalho, foram observadas maiores prevalências de TMC entre os docentes na situação de trabalho de alta exigência, seguidas das encontradas na situação de trabalho ativo. Porém, não foram encontradas associações 
estatisticamente significativas em nenhuma das situações de trabalho caracterizadas pelo modelo D-C (Tabela 6).

Tabela 6 - Prevalência (\%) de TMC, razões de prevalência e respectivos intervalos de confiança de 95\%, segundo aspectos psicossociais. Docentes universitários, Bahia, 2017

\begin{tabular}{lccccc}
\hline \multirow{2}{*}{ Aspectos Psicossociais (N) } & \multicolumn{5}{c}{ TMC } \\
\cline { 2 - 6 } & $\mathrm{n}$ & $\%$ & $\mathrm{RP}$ & IC95\% & Valor de $\mathrm{p}$ \\
\hline Modelo Demanda-Controle (123) & 6 & 18,8 & --- & -- & \\
Baixa exigência & 10 & 32,3 & 1,72 & $0,711-4,162$ & 0,21 \\
Trabalho ativo & 7 & 28,0 & 1,49 & $0,574-3,887$ & 0,40 \\
Trabalho passivo & 13 & 37,1 & 1,98 & $0,855-4,589$ & 0,09 \\
Alta exigência & & & &
\end{tabular}

Fonte: banco de dados

--- = valor de referência

Após a análise bivariada, considerando o critério adotado do valor de $\mathrm{p} \leq 0,25$, entraram para o modelo de análise multivariada 17 variáveis: idade entre 30 e 45 anos; ter filhos; local de origem outros; tempo de trabalho como docente na instituição entre 1 e 7 anos; carga horária na graduação acima de 13 horas; carga horária em atividades de pesquisa acima de 9 horas; sentimento de sobrecarga no trabalho; sentimento de pressão por publicação; sentimento de desgaste na relação com os alunos; condição regular da sala de aula; condição inadequada da sala de aula; ter sofrido agressão física ou verbal dentro do campus; não sentir segurança ou proteção dentro do campus; não sentir satisfação em trabalhar na instituição; desejo de abandonar a universidade; trabalho ativo; e trabalho de alta exigência (Tabelas 4-6).

$\mathrm{Na}$ análise multivariada permaneceram no modelo final apenas duas variáveis, as quais mantiveram associação positiva estatisticamente significativa com os TMC ( $p \leq 0,05)$ : sentir desgaste na relação com os alunos (RP: 2,31; IC: 1,367-3,914; p: 0,00) e não sentir satisfação em trabalhar na instituição (RP: 2,13; IC: 1,298-3,481; p: 0,00). As diferenças observadas foram robustas (RP superior a 2,0).

A análise diagnóstica do modelo final foi realizada por meio da curva ROC, na qual se obteve uma área igual a 0,76 abaixo da curva, demonstrando que o modelo discriminou de forma eficiente os achados finais. O teste de Hosmer-Lemeshow revelou o resultado de $\mathrm{p}=0,12$, evidenciando bom ajustamento do modelo final.

\section{Discussão}

Estudos realizados com docentes universitários brasileiros mostram resultados análogos aos encontrados nesta pesquisa em relação à idade, filhos e situação conjugal demonstrando 
similaridade no perfil docente, porém divergem quanto ao gênero que, na maior parte dos estudos, tiveram predomínio do sexo feminino (ARAÚJO et al., 2005; FERREIRA et al., 2015; FIGLIOULO; LIMA; LAURENTINO, 2011; SANTOS, 2013; SANTOS, 2016; TAVARES et $a l ., 2012)$. Destaca-se que a maior parte dos docentes tem local de origem diferente da Bahia, tal situação pode ter sido propiciada pelas oportunidades geradas pelos concursos públicos, os quais possibilitaram que docentes de várias regiões do país pudessem concorrer às vagas ofertadas.

Quanto aos aspectos laborais, a instituição estudada é relativamente jovem, porém apresenta características semelhantes a instituições mais antigas. O nível de titulação dos docentes estudados mostrou-se mais elevado do que em outros estudos envolvendo docentes universitários, destacando-se que quase a totalidade da amostra possuía doutorado e a outra parcela possuía mestrado. Nos demais estudos da literatura, os percentuais de níveis de qualificação de mestrado e doutorado foram menores (ARAÚJO et al., 2005; FERREIRA et al., 2015; SANTOS, 2016; TAVARES et al., 2012). A alta qualificação pode ser explicada pelos incentivos da universidade como a Política de Capacitação Docente que estabelece, como meta prioritária, a capacitação do quadro docente, com ênfase na qualificação e atualização sistemática, permitindo o afastamento do docente das atividades laborais por até quatro anos. Logo, os dados observados evidenciam o sucesso dessa política de capacitação. O tempo de trabalho na instituição revela um perfil do quadro docente mais experiente, com mais de oito anos de atividade na universidade. Similar ao tempo encontrado por Santos (2013) na Universidade Federal de Pernambuco (UFPE) e Borsoi (2012) na Universidade Federal do Espírito Santo (UFES), nas quais $66,3 \%$ e $63,6 \%$ dos docentes tinham mais de dez anos nas instituições, respectivamente.

Quanto às horas dedicadas às atividades laborais, observa-se que a maioria dos docentes dedica até 30 horas semanais às atividades de ensino, pesquisa, extensão e administrativas, concomitantemente, excluindo-se as outras inúmeras atividades que comumente são desempenhadas, mas que não foram computadas aqui. Tal situação explicita a pluralidade de funções que são atribuídas aos docentes no dia a dia de trabalho. Um cenário coerente com o que vem sendo observado nas instituições de ensino superior públicas, no qual a atuação do docente vai muito além do processo de ensino restrito à sala de aula. $\mathrm{O}$ cotidiano de trabalho envolve não apenas as atividades consideradas tripé da instituição universitária como ensino, pesquisa e extensão, mas também as atividades administrativas e de gestão, exigindo do docente uma postura polivalente (LEMOS, 2014). Segundo Freitas e Silva (2016), os docentes da Universidade Federal de Mato Grosso (UFMT), além de ensinar na graduação e pós-graduação, 
participam das atividades administrativas, desenvolvem pesquisas, produzem artigos, orientam alunos em diversas atividades, organizam eventos, captam recursos financeiros, ainda que não estejam preparados para assumir tais atribuições.

Com a sobreposição e acúmulo de funções, a maior parte dos docentes da instituição estudada referiu sentir sobrecarga no desempenho das atividades laborais. Tal realidade foi igualmente referida no estudo de Borsoi (2012), o qual acrescenta ainda que muitas das atividades são percebidas como burocráticas e desnecessárias, apenas um acúmulo de tarefas inócuas e desgastantes. Outro ponto importante diz respeito ao sentimento de pressão para produção e publicação de artigos, referido pelos docentes da instituição estudada, frequentemente observado nas universidades, em especial nos programas de pós-graduação, e também relatado em outros estudos (LEMOS, 2014; SANTOS, 2016). A produção científica é um meio importante para o docente se manter na pós-graduação e conseguir recursos para a realização de pesquisas, incentivando a busca incessante do aumento da produtividade e a competição entre os docentes de um mesmo programa (BORSOI, 2012).

Percentual elevado de docentes relatou não sentir desgaste na relação com os alunos, demonstrando bom relacionamento interpessoal que contribui positivamente para um bom ambiente de trabalho na universidade. No estudo conduzido na UFES, muitos docentes afirmaram que se sentiam estimulados ou realizados profissionalmente graças ao contato com os alunos, sendo esta uma relação que frequentemente trazia gratificação e prazer (BORSOI, 2012). Quanto às condições das salas de aula, de uma maneira geral, grande parte dos docentes avaliou como adequadas, resultado contraditório aos encontrados por Araújo et al. (2005) na UEFS e por Borsoi (2012) na UFES. Nesta última, mais de 2/3 dos docentes entrevistados qualificaram as condições objetivas em que as atividades docentes eram realizadas, o que inclui condições das salas de aula, como precárias.

Um dado alarmante é que parcela significativa dos docentes já sofreu alguma agressão física ou verbal, por parte de alunos, colegas de trabalho ou pessoas externas à instituição, dentro do campus da universidade. A parcela de docentes que referiu episódio de agressão foi mais que o dobro da encontrada na UFPE, na qual 20\% dos docentes referiram ter sofrido algum tipo de violência no campus (SANTOS, 2013). Este dado é extremamente preocupante e merece atenção especial tendo em vista as repercussões negativas que episódios de agressão causam na estrutura psíquica dos envolvidos. Neste contexto, a maioria dos docentes referiu não se sentir seguro ou protegido dentro da universidade, o que pode ser reflexo dos casos de agressão vivenciados ou presenciados. É necessário e urgente pensar estratégias que possam combater 
tal prática dentro da instituição, considerando o quão prejudicial é para a saúde da comunidade acadêmica o clima de insegurança e vulnerabilidade.

Importante observar que grande parte dos docentes referiu satisfação em trabalhar na universidade, porém um percentual expressivo, mais de 40\%, expressou o desejo de abandonar a instituição. O sentimento de satisfação pode estar relacionado ao prazer gerado pela profissão de docente universitário, que muitas vezes antecede experiências desagradáveis vivenciadas na instituição, como sugere Mendes et al. (2007). O desejo de abandonar a universidade estudada é um dado preocupante que deve ser investigado.

Considerando os aspectos psicossociais do trabalho, os docentes da instituição analisada tinham como características predominantes o trabalho de alta demanda psicológica e de alto controle. Considerando o modelo D-C, o maior percentual foi observado na situação de "alta exigência", enquanto que o "trabalho passivo" foi o menos frequente. Tal resultado corrobora o estudo de Figlioulo, Lima e Laurentino (2011), o qual revelou que 50,7\% dos docentes universitários apresentavam alto controle sobre o trabalho e 56,5\% alta demanda psicológica. Os autores constataram também maior frequência do trabalho de "alta exigência" $(29,5 \%)$ e menor frequência no "trabalho passivo" (20,3\%). O estudo de Tavares et al. (2012) também observou maior frequência dos docentes na situação de trabalho de "alta exigência", porém a menor frequência foi no "trabalho ativo". O resultado apresentado pelos docentes da instituição estudada é preocupante levando em conta que a principal predição do modelo D-C presume que o trabalho realizado em situação de "alta exigência" gera situações de maior repercussão negativa para a saúde mental, sendo considerado o grupo de maior exposição (ARAÚJO; GRAÇA; ARAÚJO, 2003). Assim, evidencia-se um risco aumentado de comprometimento da saúde mental na população estudada.

A presente pesquisa evidenciou expressivo comprometimento da saúde mental dos envolvidos, levando em conta que mais de 1/4 dos docentes apresentaram sinais sugestivos de TMC, identificando elevado sofrimento mental no momento de aplicação dos questionários.

A prevalência global de TMC estimada nesta pesquisa se mostrou mais elevada, comparando-se com pesquisas anteriores envolvendo docentes do ensino superior brasileiro. $\mathrm{O}$ estudo de Tavares et al. (2012) envolvendo 130 docentes dos cursos de graduação em enfermagem de sete universidades públicas federais do Rio Grande do Sul revelou que 20,1\% dos docentes apresentavam TMC. No interior da Bahia, Santos (2016) desenvolveu uma pesquisa com 340 docentes da UEFS e estimou uma prevalência de $28 \%$ de TMC. Estudo envolvendo 175 docentes do Centro de Ciências da Saúde da UFPE revelou que 17,3\% dos docentes participantes apresentavam TMC (SANTOS, 2013). Em Recife/PE, estudo 
envolvendo 69 docentes de cursos de graduação em fisioterapia de cinco instituições, uma pública e quatro privadas, encontrou prevalência de TMC de 23,2\% (FIGLIOULO; LIMA; LAURENTINO, 2011). Pesquisa realizada em instituição particular do norte de Minas Gerais com 175 docentes de nove cursos da área da saúde revelou uma prevalência de 19,5\% de TMC na população investigada (FERREIRA et al., 2015).

Em contrapartida, a prevalência de TMC encontrada neste estudo mostrou-se mais baixa quando comparada com as alarmantes prevalências apresentadas em estudos envolvendo docentes da educação básica. Rocha e Souza (2013) realizaram estudo em 31 escolas públicas municipais de Pelotas/RS, com 575 docentes do ensino médio, e estimaram a prevalência de TMC de 43,8\%. Estudo conduzido em escolas públicas e particulares de Vitória da Conquista/BA encontrou uma prevalência de 44\% em amostra de 1.024 docentes do ensino infantil e fundamental (PORTO et al., 2006). Baldaçara et al. (2015), em pesquisa envolvendo 109 docentes de escolas públicas de Palmas/TO, estimaram que 49,5\% dos docentes apresentavam TMC. Estudo com 2.133 professoras do ensino fundamental de 83 escolas da rede municipal de Belo Horizonte/MG revelou que metade da amostra, 50\%, apresentava TMC (JARDIM; BARRETO; ASSUNÇÃO, 2007).

A partir da análise multivariada evidenciou-se que, dentre os fatores analisados, sentir desgaste na relação com os alunos e não sentir satisfação em trabalhar na instituição estudada foram os fatores que se mostraram associados significantemente à ocorrência de TMC na amostra investigada.

Os docentes que referiram desgaste na relação com os alunos apresentaram prevalência de TMC 2,31 vezes maior, comparando com as apresentadas pelos docentes que referiram não sentir desgaste. Resultado similar ao encontrado por Araújo et al. (2003), em estudo envolvendo docentes de escolas particulares de Salvador/BA, no qual a força de associação entre TMC e desgaste com os alunos foi de 2,4 vezes; os docentes que referiram desgaste na relação com os alunos apresentaram prevalência de TMC de 33,1\%, os que não referiram desgaste apresentaram prevalência de 14,1\%. Em revisão sistemática, Diehl e Marin (2016) concluíram que os problemas motivacionais e comportamentais dos alunos como falta de limite, de educação e dificuldades de relacionamento estão entre os fatores responsáveis pelo adoecimento dos docentes.

A relação dos docentes com os alunos é um aspecto de crucial importância na profissão. Mendes et al. (2007), em estudo qualitativo que objetivou descrever os significados e o sofrimento na profissão de docentes da Universidade Federal de Maringá (UFM), verificaram que o contato, a convivência e a interação com os alunos são alguns dos fatores responsáveis 
pelo prazer na profissão. Por outro lado, ao mesmo tempo em que a convivência pode ser prazerosa, os desgastes oriundos desta podem ser fontes de desprazer. A falta de interesse dos alunos foi um dos aspetos relatados pelos docentes da UFM como responsáveis pelo sofrimento.

A convivência com os alunos faz parte da rotina diária de trabalho do docente e, muitas vezes, esse convívio pode ser hostil, desgastante, permeado por atritos e conflitos. Uma realidade que o docente pode não se sentir capaz de alterar, surgindo assim o sofrimento. Segundo Dejours, Abdoucheli e Jayet (2009), o trabalho patogênico ocorre quando todos os recursos defensivos foram esgotados na tentativa de transformação ou de aperfeiçoamento da organização do trabalho, levando a um sofrimento que abala o equilíbrio psíquico e que pode levar ao adoecimento. Partindo desse princípio, podemos supor que os docentes estudados, que relataram relações desgastantes com os alunos, esgotaram os seus recursos defensivos ou estes foram insuficientes para afastar a vivência de sofrimento mental. Portanto, análise de alternativas para a estruturação de relações menos conflituosas e criação de espaços nos quais se possa estabelecer comunicação e diálogo sobre os problemas enfrentados devem ser viabilizadas como forma de enfrentamento do sofrimento identificado.

Outra variável que se manteve associada aos TMC foi não sentir satisfação em trabalhar na instituição. Os docentes que relataram não sentir satisfação com o trabalho na universidade apresentaram prevalência de TMC 2,13 vezes maior, comparado aos docentes que relataram sentir satisfação.

Estudos envolvendo docentes de outros níveis de ensino corroboram tais achados. Araújo et al. (2003) avaliaram o trabalho e o sofrimento psíquico de docentes do ensino básico encontrando associação dos TMC com a insatisfação no desempenho das atividades laborais. Os autores atribuíram tal resultado à valorização profissional percebida e autoestima. Jacarandá (2008) em estudo sobre o sofrimento mental e sua correlação com a satisfação no trabalho, envolvendo docentes de escolas inclusivas estaduais de Porto Velho/RO, verificou correlação significativa entre as variáveis satisfação no trabalho e TMC inversamente proporcional; ou seja, quanto mais elevada a satisfação no trabalho, menor a prevalência de TMC.

A satisfação e insatisfação no trabalho foram abordadas em outros estudos envolvendo docentes universitários. Borsoi (2012) atribuiu o sentimento de insatisfação ao final da jornada de trabalho ao cansaço e esgotamento referidos pelos docentes da UFES. Leite et al. (2008), em estudo com docentes da Universidade Federal de Ouro Preto, verificaram baixo nível de satisfação dos docentes e relacionaram a insatisfação no trabalho com o estresse. Para estes autores, os descontentamentos do trabalhador associados à impossibilidade de mudanças das questões que não o satisfazem levam ao desgaste no ambiente de trabalho. 
O sofrimento mental do trabalhador decorre de aspectos presentes na organização do trabalho que atuam no sentido de reduzir os espaços de "comportamento livre", entendido como um padrão comportamental composto pela tentativa de transformação da realidade, conforme os desejos do trabalhador. Quanto mais a organização do trabalho inibe a manifestação dos comportamentos livres, mais restritas ficam as possibilidades de resolução dos conflitos entre os desejos do trabalhador e as demandas do trabalho, gerando insatisfação e sofrimento. O sofrimento gerado constitui a porta de entrada para o adoecimento psíquico (DEJOURS, 1992).

Mendes et al. (2007) observaram que assim como são inúmeros os motivos de satisfação e prazer no trabalho relatados pelos docentes da UEM, também são numerosos os motivos de insatisfação e desprazer; e acrescentam que tais motivos podem estar relacionados tanto à instituição diretamente como às relações oriundas desta. Dentre os motivos citados como de satisfação estão transmissão de conhecimento, possibilidade de aprender coisas novas, contato com alunos e flexibilidade de horários; dentre os motivos de insatisfação estão excesso de burocracia, defasagem do salário e falta de interesse dos alunos. Para Borsoi (2012), a sobrecarga laboral, as condições inadequadas de trabalho, a jornada diária de trabalho sem uma delimitação clara de tempo e a pressão pela produtividade formam um conjunto de fatores que conformam uma percepção negativa do trabalho docente.

Nesta pesquisa não foi possível identificar os motivos responsáveis pelo sentimento de desgaste na relação com os alunos e nem da falta de satisfação, posto não ter sido objeto de estudo. Porém, considerando a subjetividade destes aspectos e suas expressivas associações com os TMC, torna-se importante aprofundar a investigação dos motivos que produzem o sofrimento psíquico observado, objetivando reduzir os desgastes e aumentar a satisfação.

É necessário considerar a presença de alguns vieses e limitações no presente estudo. Cabe mencionar que apesar da taxa de resposta ter sido considerada muito boa, $79,9 \%$, os resultados obtidos podem não refletir a realidade da instituição estudada, uma vez que se considerou apenas uma unidade, dentre as cinco existentes. Tais resultados, portanto, expressam a situação dos docentes participantes da pesquisa, considerando que não foi aplicada amostragem aleatória, não cabendo inferência estatística. $\mathrm{O}$ tamanho amostral também pode ter sido insuficiente para avaliação da importância de algumas variáveis, o que pode ter comprometido a análise. Além disso, é preciso considerar que com o tipo de desenho de estudo adotado, corte transversal, não é possível estabelecer a relação de causa e efeito, na medida em que a análise da exposição e do desfecho é realizada concomitantemente. Existe assim, a possibilidade de causalidade reversa, não podendo ser determinado com precisão se os fatores associados levaram aos TMC ou se foram os TMC que levaram aos fatores associados. Outro 
aspecto a considerar diz respeito ao "efeito do trabalhador sadio", presente nos estudos transversais de grupos ocupacionais, no qual são incluídos apenas os trabalhadores que "sobreviveram" à doença em estudo. Apesar dos TMC não serem fatais, foram selecionados para participar do estudo os docentes em efetivo exercício e foram excluídos aqueles que abandonaram a instituição ou que estavam afastados ou licenciados à época da pesquisa. Como estes podem representar uma amostra importante para o estudo, há a possibilidade de perda de informações que podem gerar subestimação dos dados. Ainda, é necessário levar em conta o tipo de instrumento utilizado, um questionário autopreenchível baseado em questões subjetivas. Portanto, deve-se ponderar a possibilidade do viés de resposta ao serem analisadas medidas autorrelatadas pelos docentes.

\section{Conclusão}

O presente estudo envolvendo docentes universitários de uma universidade pública do interior da Bahia estimou a prevalência de TMC de 29,9\%. Um dado preocupante que demanda atenção, considerando que mais de 1/4 da população estudada apresentou sinais sugestivos de sofrimento mental. Tal quadro pode trazer danos não apenas para o docente diretamente, mas também para a instituição, levando em conta a possibilidade de comprometimento da qualidade de ensino e das relações interpessoais, além dos prováveis afastamentos que geram prejuízos e custos para a instituição.

A partir da análise estatística, verificou-se associação positiva estatisticamente significante entre TMC e os sentimentos de desgaste na relação com os alunos e de insatisfação em trabalhar na instituição. Diante de tal cenário, alguns aspectos são passíveis de intervenção por parte da instituição como, por exemplo, a busca pela melhoria das condições e organizações do trabalho na universidade a fim de melhorar a relação dos docentes com os alunos, assim como o grau de satisfação destes profissionais em trabalhar na instituição. Para tanto, é importante investigar quais fatores estão efetivamente relacionados aos sentimentos de desgaste e de insatisfação dos docentes por meio de pesquisa qualitativa, considerando o caráter subjetivo de tais aspectos.

A instituição estudada possui curso de graduação em Psicologia, assim, sugere-se pensar na possibilidade de implantação de um serviço de assistência e acompanhamento psicológico voltado ao docente. Do mesmo modo, rodas de conversa, palestras informativas, atividades integrativas e diversos eventos podem ser realizados a fim de proporcionar espaço de trocas e 
apoio, visando minimizar os impactos negativos que o cotidiano de trabalho na universidade pode trazer para a saúde mental destes profissionais.

Além disso, o baixo custo e a facilidade de aplicação do questionário, utilizado neste estudo, viabilizam ampliar a investigação a toda comunidade acadêmica da instituição servidores e alunos. Desta forma, sugere-se estender este estudo às demais unidades e agentes da universidade estudada objetivando alcançar um diagnóstico geral da instituição.

\section{Referências}

ARAÚJO, Tânia Maria de et al. Trabalho docente e sofrimento psíquico: um estudo entre professores de escolas particulares de Salvador, Bahia. Revista da FAEEBA: Educação e Contemporaneidade, Salvador, v. 12, n. 20, p. 485-495, jul./dez., 2003.

ARAÚJO, Tânia Maria de et al. Mal-estar docente: avaliação de condições de trabalho e saúde em uma instituição de ensino superior. Revista Baiana de Saúde Pública, Salvador, v. 29, n. 1, p. 6-21, jan./jun. 2005.

ARAÚJO, Tânia Maria de; CARVALHO, Fernando Martins. Condições de trabalho docente e saúde na Bahia: estudos epidemiológicos. Educação e Sociedade, Campinas, v. 30, n. 107, p. 427-449, maio/ago. 2009.

ARAÚJO, Tânia Maria de; GRAÇA, Cláudia Cerqueira; ARAÚJO, Edna. Estresse ocupacional e saúde: contribuições do modelo Demanda-Controle. Ciência e Saúde Coletiva, Rio de Janeiro, v. 8, n. 4, p. 991-1003, 2003.

BALDAÇARA, Leonardo et al. Common psychiatric symptoms among public school teachers in Palmas, Tocantins, Brazil. An observational cross-sectional study. São Paulo Medical Journal, São Paulo, v. 133, n. 5, p. 435-438, set./out. 2015.

BEUSENBERG, Michale et al. A user's guide to the Self Reporting Questionnaire (SRQ). Geneva: Division of Mental Health, World Health Organization, 1994.

BORSOI, Izabel Cristina Ferreira. Trabalho e produtivismo: saúde e modo de vida de docentes de instituições públicas de ensino superior. Cadernos de Psicologia Social do Trabalho, São Paulo, v. 15, n. 1, p. 81-100, jun. 2012.

CARLOTTO, Mary Sandra; CÂMARA, Sheila Gonçalves. Prevalence and risk factors of common mental disorders among teachers. Journal of work and organizational psychology, Madri, v. 31, n. 3, p. 201-206, 2015.

DEJOURS, Christophe. A loucura do trabalho: estudo de psicopatologia do trabalho. 5. ed. São Paulo: Cortez-Oboré, 1992. 
DEJOURS, Christophe; ABDOUCHELI, Elisabeth; JAYET, Christian. Psicodinâmica do trabalho: contribuições da Escola Dejouriana à análise da relação prazer, sofrimento e trabalho. São Paulo: Atlas, 2009.

DELCOR, Núria Serre et al. Condições de trabalho e saúde dos professores da rede particular de ensino de Vitória da Conquista, Bahia, Brasil. Cad. Saúde Pública, Rio de Janeiro, v. 20, n. 1, p. 187-196, jan./fev. 2004.

DIEHL, Liciane; MARIN, Angela Helena. Adoecimento mental em professores brasileiros: revisão sistemática da literatura. Estudos Interdisciplinares em Psicologia, Londrina, v. 7, n. 2, p. 64-85, dez. 2016.

FERREIRA, Raquel Conceição et al. Transtorno mental e estressores no trabalho entre professores universitários da área da saúde. Trabalho, educação e saúde, Rio de Janeiro, v. 13, p. 135-155, 2015.

FIGLIOULO, Danielle Santana da Silva; LIMA, Pedro Olavo de Paula; LAURENTINO, Glória Elizabeth Carneiro. Estresse ocupacional e fadiga em fisioterapeutas que exerciam função de docência em universidades da cidade de Recife/PE. Terapia Manual, [S. L.], v. 9, n. 43, p. 231-237, 2011.

FREITAS, Paula Adriana Lima de Matos; SILVA, Marluce Souza e. Trabalho docente cercado por circunstâncias que são fontes de adoecimento. Revista Direitos, trabalho e política social, Cuiabá, v. 2, n. 2, p. 126-151, jan./jun., 2016.

GLINA, Débora Miriam Raab et al. Saúde mental e trabalho: uma reflexão sobre o nexo com o trabalho e o diagnóstico, com base na prática. Cad. Saúde Pública, Rio de Janeiro, v. 17, n. 3, p. 607-616, maio/jun., 2001.

GONÇALVES, Daniel Maffasioli; STEIN, Airton Telelbon; KAPCZINSKI, Flavio. Avaliação de desempenho do Self-Reporting Questionnaire como instrumento de rastreamento psiquiátrico: um estudo comparativo com o Structured Clinical Interview for DSM-IV-TR. Cad. Saúde Pública, Rio de Janeiro, v. 24, n. 2, p. 380-390, 2008.

JACARANDÁ, Elza Maria de Freitas. Sofrimento mental e satisfação no trabalho: um estudo com professores das escolas inclusivas estaduais de ensino fundamental em Porto Velho, Rondônia. 96f. Dissertação (Mestrado Ciências da Saúde) - Faculdade de Ciências da Saúde, Universidade de Brasília, Brasília, 2008.

JARDIM, Renata; BARRETO, Sandhi Maria; ASSUNÇÃO, Ada Ávila. Condições de trabalho, qualidade de vida e disfonia entre docentes. Cad. Saúde Pública, Rio de Janeiro, v. 23, n. 10, p. 2439-2461, out. 2007.

LEITE, Délia Ribeiro et al. Trabalho docente em foco: relação entre as condições de trabalho e o adoecimento de professores na Universidade Federal de Ouro Preto. Trabalho e Educação, Belo Horizonte, v. 17, n. 3, p. 71-83, set./dez. 2008.

LEMOS, Denise Vieira da Silva. Precarização do trabalho docente nas Federais e os impactos na saúde: o professor no seu limite. Entreideias, Salvador, v. 3, n. 1, p. 95-109, jan./jun. 2014. 
LUDERMIR, Ana Bernarda; MELO FILHO, Djalma A. de. Condições de vida e estrutura ocupacional associadas a transtornos mentais comuns. Rev. Saúde Pública, São Paulo, v. 36, n. 2, p. 213-221, 2002.

LYRA, Gabriela Franco Dias et al. A relação entre professores com sofrimento psíquico e crianças escolares com problemas de comportamento. Ciência e Saúde Coletiva, Rio de Janeiro, v. 14, n. 2, p. 435-444, 2009.

MENDES, Luciano et al. Da arte ao ofício: vivências de sofrimento e significado do trabalho de professor universitário. Rev. Mal-Estar Subj., Fortaleza, v. 7, n. 2, p. 527-556, set. 2007.

PORTO, Lauro Antonio et al. Associação entre distúrbios psíquicos e aspectos psicossociais do trabalho de professores. Revista de Saúde Pública, São Paulo, v. 40, n. 5, p. 818-826, 2006.

REIS, Eduardo José Farias Borges dos et al. Trabalho e distúrbios psíquicos em professores da rede municipal de Vitória da Conquista, Bahia, Brasil. Cad. Saúde Pública, Rio de Janeiro, v. 21, n. 5, p. 1480-1490, set./out. 2005.

ROCHA, Luise Marques da; SOUZA, Luciano Dias de Mattos. Voice Handicap Index associated with Common Mental Disorders in elementary school teachers. Journal of voice, v. 27, n. 5, p. 595-602, 2013.

SANTOS, Kionna Oliveira Bernardes et al. Avaliação de um instrumento de mensuração de morbidade psíquica: estudo de validação do Self-Reporting Questionnaire (SRQ-20). Revista Baiana de Saúde Pública, Salvador, v. 34, n. 3, p. 544-560, jul./set. 2010.

SANTOS, Gustavo Barreto. Bem estar e condições de trabalho de professores do Centro de Ciências da Saúde da Universidade Federal de Pernambuco. 71f. Dissertação (Mestrado em Saúde Coletiva) - Centro de Ciências da Saúde, Universidade Federal de Pernambuco, Recife, 2013.

SANTOS, Daniel Alberto Santos. Estresse ocupacional e transtornos mentais comuns entre professores universitários. 157f. Dissertação (Mestrado em Saúde Coletiva) Departamento de Saúde, Universidade Estadual de Feira de Santana, Feira de Santana, 2016.

SANTOS, Kionna Oliveira Bernardes; ARAÚJO, Tânia Maria de; OLIVEIRA, Nelson Fernandes de. Estrutura fatorial e consistência interna do Self-Reporting Questionnaire (SRQ20) em população urbana. Cad. de Saúde Pública, Rio de Janeiro, v. 25, n. 1, p. 214-222, jan. 2009.

SANTOS, Élem Guimarães dos; SIQUEIRA, Marluce Miguel de. Prevalência dos transtornos mentais na população adulta brasileira: uma revisão sistemática de 1997 a 2009. Jornal

Brasileiro de Psiquiatria, Rio de Janeiro, v. 59, n. 3, p. 238-246, 2010.

SILVA, Luciane Goulart da; SILVA, Marcelo Cozzensa da. Condições de trabalho e saúde de professores pré-escolares da rede pública de ensino de Pelotas, RS, Brasil. Ciência e Saúde Coletiva, Rio de Janeiro, v. 18, n. 11, p. 3137-3146, nov. 2013. 
SILVA-JUNIOR, João Silvestre; FISCHER, Frida Marina. Afastamento do trabalho por transtornos mentais e estressores psicossociais ocupacionais. Rev. bras. epidemiol., São Paulo, v. 18, n. 4, p. 735-744, out./dez. 2015.

TAVARES, Juliana Petri et al. Distúrbios psíquicos menores em enfermeiros docentes de universidades. Rev. Latino-Am. Enfermagem, Ribeirão Preto, v. 20, n. 1, jan./fev. 2012. 